\title{
Anti-CD137 Agonistic Monoclonal Antibody CTX-471
}

National Cancer Institute

\section{Source}

National Cancer Institute. Anti-CD137 Agonistic Monoclonal Antibody CTX-471. NCI

Thesaurus. Code C162039.

A fully human immunoglobulin G4 (IgG4) agonistic monoclonal antibody targeting CD137 (4-1BB; tumor necrosis factor receptor superfamily member 9; TNFRSF9), with potential immunostimulating and antineoplastic activities. Upon administration, anti-CD137 agonistic monoclonal antibody CTX-471 binds to and activates CD137 expressed on a variety of leukocyte subsets including activated T-lymphocytes and natural killer (NK) cells. This enhances CD137-mediated signaling, induces cytokine production and promotes T-cell mediated anti-tumor immune responses. CD137, a surface glycoprotein of the tumor necrosis factor receptor superfamily, is an inducible costimulatory receptor that plays a key role in T-cell proliferation, survival and cytolytic activity. 